\title{
Preparation of an Epoxy/Polyaniline Composite Coating and Its Passivation Effect on Cold Rolled Steel
}

\author{
Yangyong WANG and Xinli JING ${ }^{\dagger}$ \\ School of Environmental and Chemical Engineering, Xi'an Jiaotong University, \\ Xi'an, 710049, People's Republic of China
}

(Received September 24, 2003; Accepted February 26, 2004; Published May 15, 2004)

\begin{abstract}
Aniline (ANI) was polymerized with the presence of solid epoxy resin (EP) powder through common oxidation polymerization and polyaniline (PANI) coated EP particles, that is a core-shell structure of PANI and EP, were achieved. Dedoped with aqueous ammonia, emeraldine base form (EB) coated EP particles (EP/EB) were obtained also. The particles were ground with an appropriate solvent by ball milling and a uniform dispersion of epoxy and nano PANI was obtained. The dispersion was formulated with curing agent to coat cold rolled steel (CRS) and the passivation effect was proved by electrochemical tests. All the EP/EB beared much higher yields compared to the pure EB polymerized under the same condition. UV-vis spectra indicate EB in EP/EB possess a lowered oxidation states with the decrease of ANI/EP in the reactants. Both UV-vis and XPS analyses indicate the existence of H-bonding between EB and EP molecules. All EP/EBs exhibit similar thermal behaviors to EP due to the extremely low EB loading. Corrosion potential measurements show that EB coating, which was prepared firstly by ball milling of EP/EB particles and then mixed and cured with a curing agent, provides excellent anti-corrosive effect for cold rolled steel (CRS) in extremely low EB loading, which comes from the nano-dispersed EB particles in the EB coatings attested by TEM. [DOI 10.1295/polymj.36.374]

KEY WORDS Polyaniline / Epoxy Resin / Corrosion Protection / Coating /
\end{abstract}

Polyaniline (PANI) is one of the mostly investigated intrinsic electric conductive polymers for its promising potential applications, such as corrosion protection, antistatic materials, ${ }^{1-3}$ plastic light-emitting diodes, ${ }^{4}$ gas or liquid separation ${ }^{5}$ and so on, also for its environmental stability and ease of preparation. PANI is built up from reduced $\left(\longrightarrow-{ }^{-\mathrm{NH}}-\right.$ and oxidized $(\square-\mathrm{N}=\mathrm{N}+$ repeat units and whose electronic properties can be reversibly controlled by protonation. The different ratios of amine to imine units yield various structures like the reduced form leucoemeraldine, the half oxidized form emeraldine base (EB) and the fully oxidized form pernigraniline.

On the other hand, PANI are insoluble in common organic solvents due to the extensive delocalization of $\pi$ electrons. Although many works have been done to make PANI soluble in $N$-methyl pyrrolidnone (NMP), dimethyl sulfoxide (DMSO) and dimethyl formamide (DMF) using functionalized organic acids or other dopants, ${ }^{6,7}$ also with the help of alkyl substituted PANI ${ }^{8}$ the results are unsatisfactory for its successful commercialization. In addition to the way of preparing soluble PANI, dispersion of PANI in traditional polymers is another useful method for PANI's researches and applications. For example, EB particles dispersed in epoxy resin coatings by our group exhibited good corrosion property with a necessary EB loading of $c a .10 \mathrm{wt} \%$. However, as indicated in refer- ence, ${ }^{9}$ distance of particles randomly dispersed in a polymer matrix decreases with decreasing particle size at the same volume fraction. Whereas, when a bulk solid was changed into smaller particle, a colloidal dispersion, the surface area (and the interfacial area between the dispersed phase and the dispersing medium) was magnified according to equation: ${ }^{10}$ Area/Mass $=6 / \rho d$ (in which $\rho$ is the density of the solid and $d$ the diameter of the particle). Therefore, it is always necessary to invest a certain significant amount of energy to create dispersions, which are systems in a state far from equilibrium. Under isothermal conditions the necessary energy is equal to the energy needed for creating new surfaces from the bulk (of both components, the dispersed phase and the dispersing medium), therefore the size of the final dispersed granule was restricted by the energy put on by mechanical stirring, though vigorously, or shuffle. ${ }^{11}$

Many papers have deposited PANI on surfaces such as fibers, ${ }^{12}$ glass textile, ${ }^{13}$ polystyrene,${ }^{14}$ glass ${ }^{15}$ and platinum, ${ }^{16}$ montmorillonite ${ }^{17}$ etc. and it is possible to choose or adjust the reaction conditions to make the polymerization proceeding preferably on the treated surface and obtain a composite with thin PANI film.

In this paper, we presented the chemical oxidation of aniline with ammonium peroxydisulfate (APS) in the presence of solid epoxy resin granules and dis-

${ }^{\dagger}$ To whom correspondence should be addressed (E-mail: xljing@mail.xjtu.edu.cn). 
persed the composite granules in a solvent to make nano-dispersed EB film. We also evaluated the anticorrosive effectiveness of the EB containing films by means of corrosion potential measuring.

\section{EXPERIMENTAL}

\section{Synthesis of the Composite Granules}

Aniline was oxidized with APS in the presence of dispersion of epoxy resin granules in aqueous hydrochloric acid. Freshly distilled aniline was dissolved in $1 \mathrm{~mol} / \mathrm{dm}^{3}$ hydrochloric acid of a beaker of certain volume and brought to the desired temperature $\left(20^{\circ} \mathrm{C}\right)$ with magnetic stirring. The powders with a granule diameter of $c a .500 \mu \mathrm{m}$, which are of solid bisphenol-A epoxy resin with an epoxy value of $0.20 \mathrm{~mol} / 100 \mathrm{~g}$, were dispersed in abovementioned aniline hydrochloride and stirred vigorously for another $20 \mathrm{~min}$. Afterwards, APS solution of hydrochloric acid maintained the same temperature was added drop wise to start the polymerization. Molar ratio of aniline to APS was fixed at 1 in all experiments. Aniline content was varied with different concentrations based on initial weight of dispersed granules. After a few minutes, the color of the dispersion turns to green and then dark green. Another period of $1.5 \mathrm{~h}$ was needed to complete the reaction, and then the product was collected on a Büchner funnel and washed several times with distilled water until the filtrate became colorless. The synthesized composite granules were further treated with $5.0 \mathrm{wt} \%$ ammonia water for $1.5 \mathrm{~h}$ and then washed to neutral with deionized water. Isolated composite granules were then dried at ambient temperature to obtain emeraldine base form of polyaniline (EB) coated epoxy resin and denoted as EP/EB.

\section{Calculation of the Yields and PANI Contents}

The yield was defined as $Y=W_{\mathrm{c}} /\left(W_{\mathrm{e}}+W_{\mathrm{m}}\right)$, where $W_{\mathrm{c}}$ is the weight of the composite granules, $W_{\mathrm{e}}$ the weight of the epoxy and $W_{\mathrm{m}}$ the weight of the aniline monomers. The PANI content in the composite granules was defined as $C=\left(W_{\mathrm{c}}-W_{\mathrm{e}}\right) / W_{\mathrm{c}}$.

\section{Preparation of Coatings}

EP/EB prepared above was added to methyl isobutyl ketone (MIBK) as designed weight ratio and ground in a planetary ball mill (QM-1SP 2 L, Nanjing
University, China) with ceramic grinding media for $1.5 \mathrm{~h}$ at a rotate speed of 500 rounds per minute. A dispersion of EB was obtained and mixed with an aromatic curing reagent (T-31, Wuxi Resin Factory, Epoxy resin/T-31 = 4.0 (weight)) whereafter. Following curing, EB coatings were prepared.

\section{Morphological and Structural Characterization}

EP/EB particles were characterized by a S-2700 scanning electron microscope (TEM). UV-vis spectra were recorded in a UV-1100 spectrophotometer and FT IR spectra were obtained from $\mathrm{KBr}$ pellets using PerkinElmer 1760 spectrophotometer. X-Ray photoelectron spectra (XPS) were recorded with PHI 5400 ESCA spectrometer using Mg K $\alpha$ X-Rays. Thermal analyses were performed on STA409PC multi-thermoanalyzer (NETZSCH) with a heating rate of $10^{\circ} \mathrm{C} \mathrm{min}^{-1}$ under nitrogen atmosphere. EB coatings were subjected to transmission electron microscopy (TEM) study after completely cured, using a JEM2000CX transmitting electron microscope.

\section{Corrosion Tests}

EB coatings were coated on cold rolled steel (CRS) plates $(100 \mathrm{~mm} \times 20 \mathrm{~mm} \times 1 \mathrm{~mm})$, which were well polished with emery cloth and degreased in acetone, and all-edge sealed with a mixture of paraffin and rosin (equal weight) to prepare working electrode. Corrosion potential, which was taken as the equilibrium open circuit potential of the testing system, was measured using a conventional three-electrode electrochemical cell with a platinum counter electrode and a saturated $\mathrm{Ag} / \mathrm{AgCl}$ reference electrode on a DJS292 potentiostat (Leici Instrument Factory, Shanghai, China) at room temperature. The corrosion medium was $3.0 \mathrm{wt} \%$ aqueous $\mathrm{NaCl}$.

\section{RESULTS AND DISCUSSION}

\section{Yield}

The feed ratios of granular epoxy to aniline and all the yields were listed in Table I. It can be seen that yields were far larger than that of pure PANI synthesized under the same condition, which was about $78 \%{ }^{18}$ The higher yields were mostly come from the fixedness of epoxy resin weight which was the main part compared to the amount of aniline before

Table I. The feed ratios and yields

\begin{tabular}{lccccc}
\hline & A & B & C & D & E \\
\hline Epoxy/ANI (wt) & 26.41 & 35.58 & 46.57 & 66.66 & 114.93 \\
\hline Yield (\%) & $98.2 \pm 0.5$ & $99.1 \pm 0.5$ & $98.5 \pm 0.5$ & $97.9 \pm 0.5$ & $98.7 \pm 0.5$ \\
\hline EB contents in EP/EBs (\%) & 2.73 & 2.05 & 1.58 & 1.11 & 0.66 \\
\hline EB contents in EB coatings (\%) & 2.21 & 1.65 & 1.27 & 0.89 & 0.53 \\
\hline
\end{tabular}




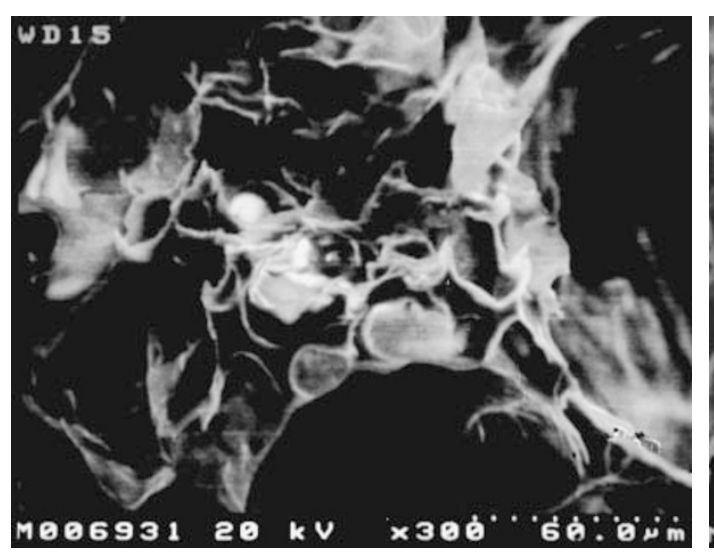

(A) Sample A

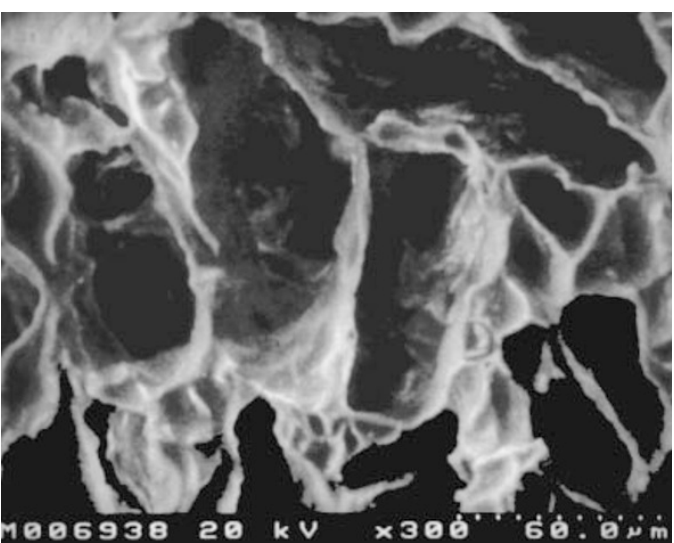

(B) Sample B

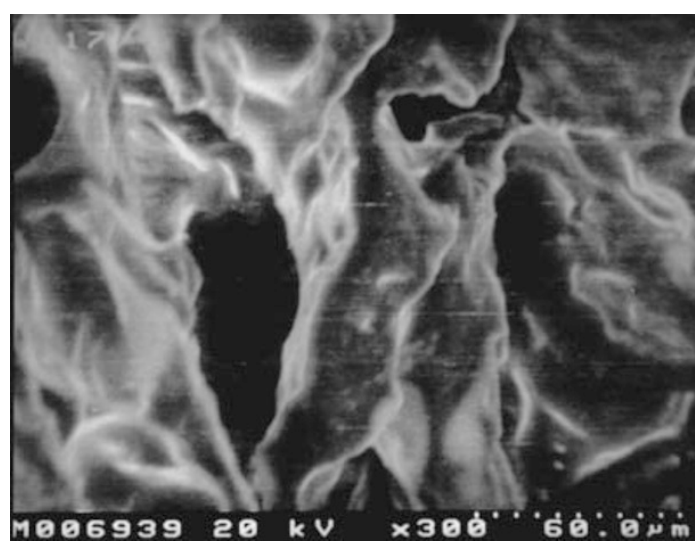

(C) Sample C

Figure 1. Morphology of EB in extracted EP/EB particles.

and after the synthesis, or to say the higher yields were benefited from a mathematical calculation. Errors of \pm 0.5 appended to the yields was rendered as the errors engendered from the experiments, including weighing and measuring process.

\section{Morphology}

The EP/EB composite particles were extracted in a Soxhlet with dimethylbenzene until EP was extracted completely and the EB left after extraction was dried and studied by SEM. The shell structure of EB was observed clearly with the EP core be extracted off (Figure 1). Thickness of the EB shell decreased with the decrease of ANI in the reaction media. The thin EB shell in Sample D and E were too weak to stand the strike of dimethylbenzene while extracting and no SEM photo was obtained.

\section{Spectral Analyses}

UV-vis spectra (Figure 2) of the EP/EB composite particles were measured by dissolving the particles in DMF. As one can see, all of the particles have two major absorption around $320 \mathrm{~nm}$ and $630 \mathrm{~nm}$, which are related to the $\pi-\pi^{*}$ and $n-\pi^{*}$ transitions of the qui-

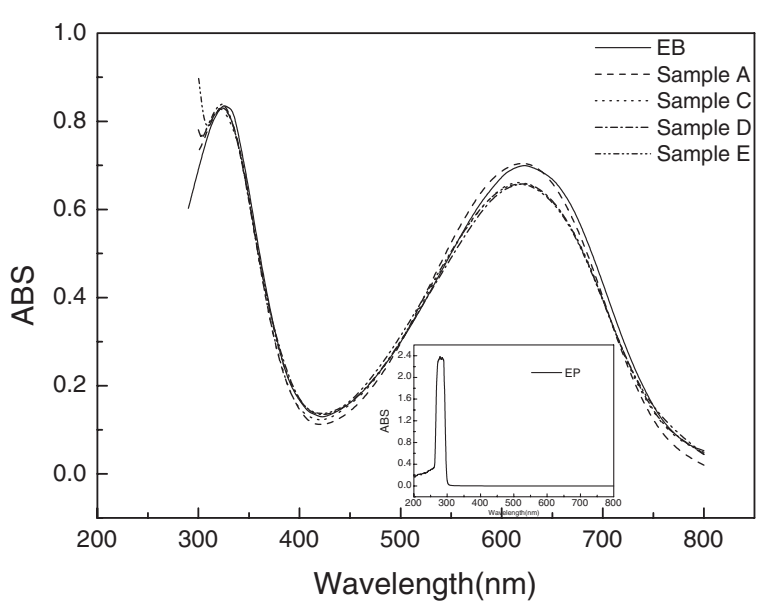

Figure 2. UV-vis spectra of EP/EB particles in DMF.

noid and benzenoid segments respectively. ${ }^{19} \mathrm{EP}$ itself having no specific absorption in the particular wavelength region of $300-800 \mathrm{~nm}$ (inset in Figure 2), the optical properties of the composites are solely for the EB component. The intensity of the $630 \mathrm{~nm}$ absorption band decreases with the ANI in the reaction media, which indicates the EB in EP/EB particle pos- 


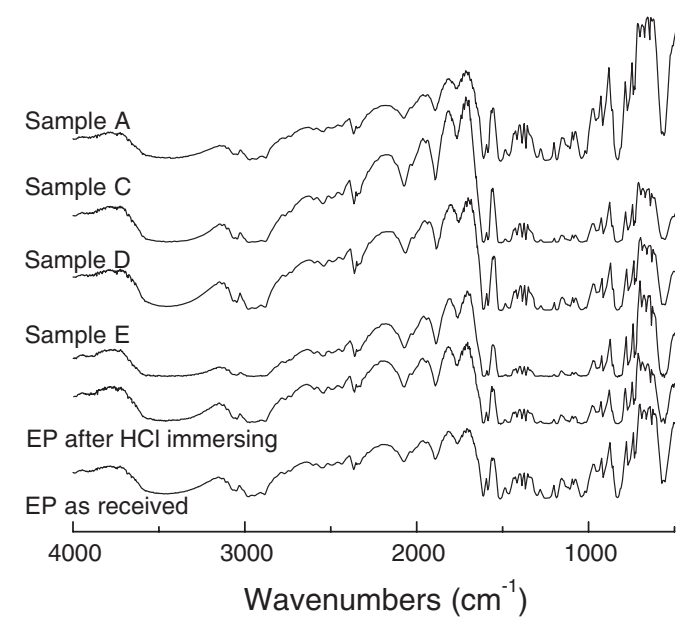

Figure 3. FT IR spectra of EP and EP/EB particles.

sesses a lower oxidation states. Furthermore, a hypsochromic shift from $626 \mathrm{~nm}$ for pure EB to $619 \mathrm{~nm}$ for Sample D and E observed implying notable engagement of amine (-NH-) groups of the benzenoid segments of EB in establishing significant degree of intermolecular H-bonding mostly utilizing the epoxy groups of EP.

The EP particles showed no distinct changes in FT IR spectra (Figure 3) after 3 h's immersion in $1 \mathrm{~mol} /$ $\mathrm{dm}^{3}$ hydrochloric acid and then rinsed with deionized water and dried, indicating that the in-situ polymerization medium have no minus effect on the EP particles. The bands of 1593 and $1506 \mathrm{~cm}^{-1}$ are corresponding to the quinoid and benzenoid ring-stretching modes of EB. The band at $3460 \mathrm{~cm}^{-1}$ is assignable to the $\mathrm{O}-\mathrm{H}$ stretch; bands fell in the range of 2880$2960 \mathrm{~cm}^{-1}$ to the $\mathrm{C}-\mathrm{H}$ stretch in $\mathrm{CH}_{2}$ and $\mathrm{CH}_{3}$; bands of 1510,1580 and $1608 \mathrm{~cm}^{-1}$ to the aromatic $\mathrm{C}=\mathrm{C}$ stretch; and a $820 \mathrm{~cm}^{-1}$ band to an aromatic $\mathrm{C}-\mathrm{H}$ out-of-plane bending. Generally speaking, the FT IR spectra of all the EP/EB particles are nearly the same and the bands at 1250, 1040, 915, 575-555, 1380 and $1360 \mathrm{~cm}^{-1}$ are due to the bisphenol-A epoxy resin.

\section{XPS Analyses}

PANI synthesized in similar reaction condition to that of $\mathrm{EP} / \mathrm{EB}$ is in the emeraldine base oxidative state, which is indicated by the equal percentage of $N_{\text {imine }}$ and $N_{\text {amine }}$ in XPS analyses. The percentage of $N_{\text {amine }}$ in EP/EB particles decreased with the increase of EP particles in the syntheses reaction medias. In the case of Sample C and E (Figure 4), the $N_{\text {amine }}$ percentages were of $42.63 \%$ and $28.83 \%$, respectively. The decrease relative to the EB could be indicating that EP interacts with EB through the formation hydrogen bonds between the amine groups in EB and epoxy groups presented in EP as suggested in Figure 5. And the remaining $N_{\mathrm{s}}(2.52 \%$ and

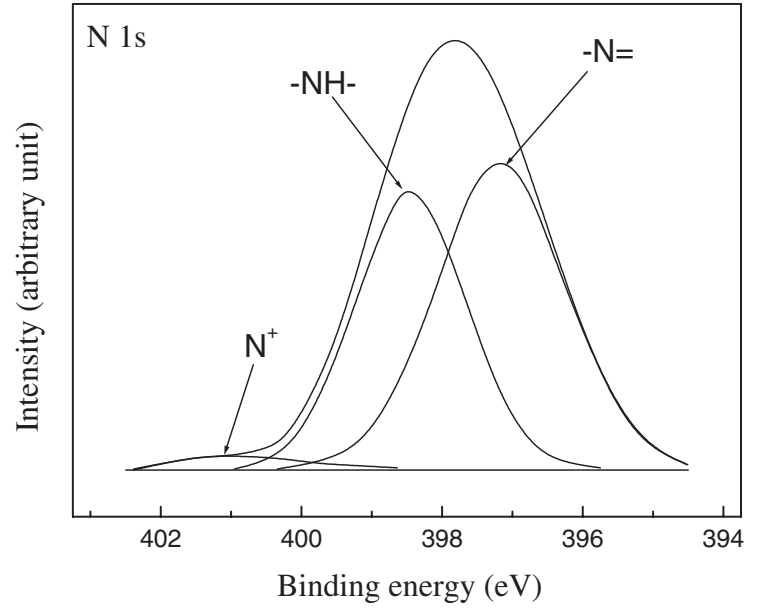

(A)

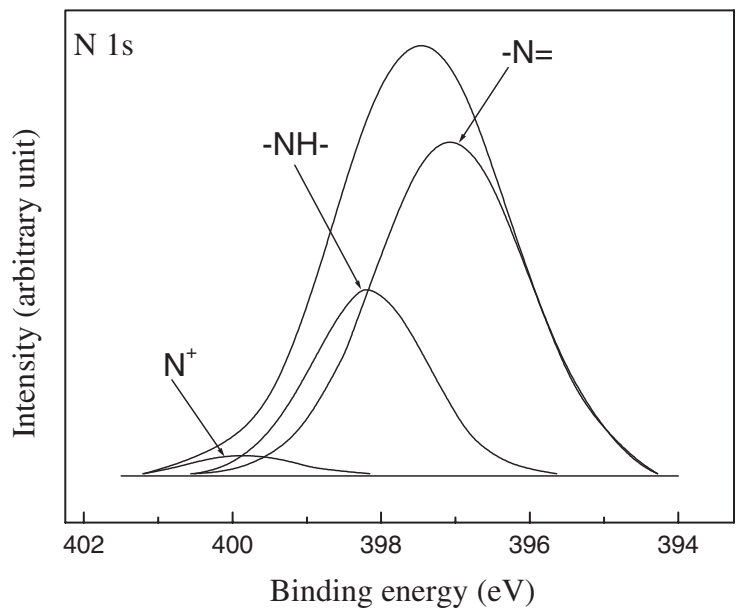

(B)

Figure 4. XPS spectra (N 1s) for (A) Sample C and (B) Sample E.

$2.42 \%$ for Sample C and D respectively) are attributed to the $\mathrm{N}^{+}$formation which are due to the surface partial oxidation or of oxygen atoms weakly complexed. ${ }^{20}$ The different positions of $\mathrm{N}^{+}$in XPS spectra of Sample C and E were probably due to the different surface oxidation extent or oxygen complexation.

\section{Thermogravimetry}

In general, the thermal behavior of the pure doped PANI shows a three-step weight loss process. ${ }^{21}$ The first weight loss below $100^{\circ} \mathrm{C}$ is attributed to the loss of water and the second weight loss ranging from 200 to $300^{\circ} \mathrm{C}$ is believed to be due to the elimination of acid dopant, for example $\mathrm{HCl}$. The third weight loss starting at around $400^{\circ} \mathrm{C}$ is assigned to the thermal decomposition of PANI backbone chains. ${ }^{22}$ TGA curves for $\mathrm{EB}$ and EP/EB particles are shown in Figure 6. The thermal degradation for EB can be roughly divided into two main weight-loss steps. The first weight loss of $c a .5 \%$ from room temperature to $100^{\circ} \mathrm{C}$ is due primarily to the expulsion of absorbed water; 


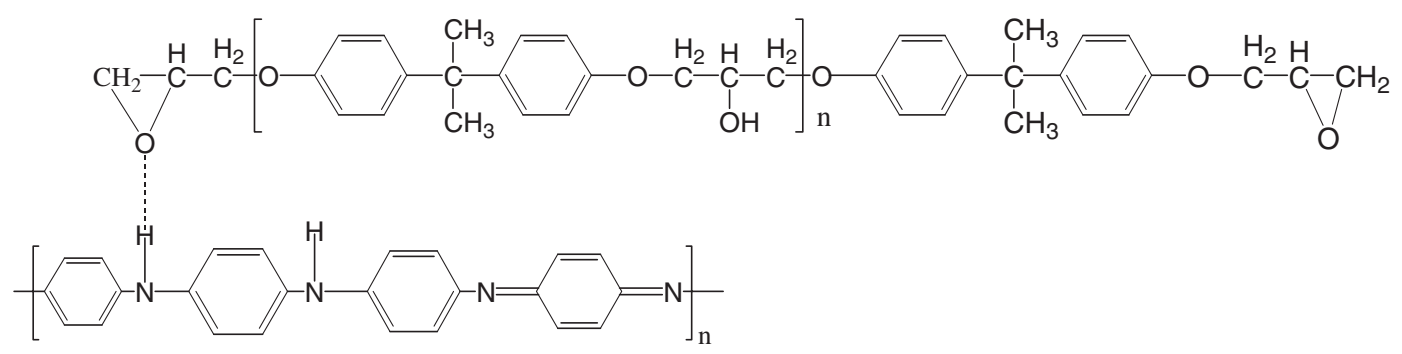

Figure 5. Hydrogen bonding between EB and EP.

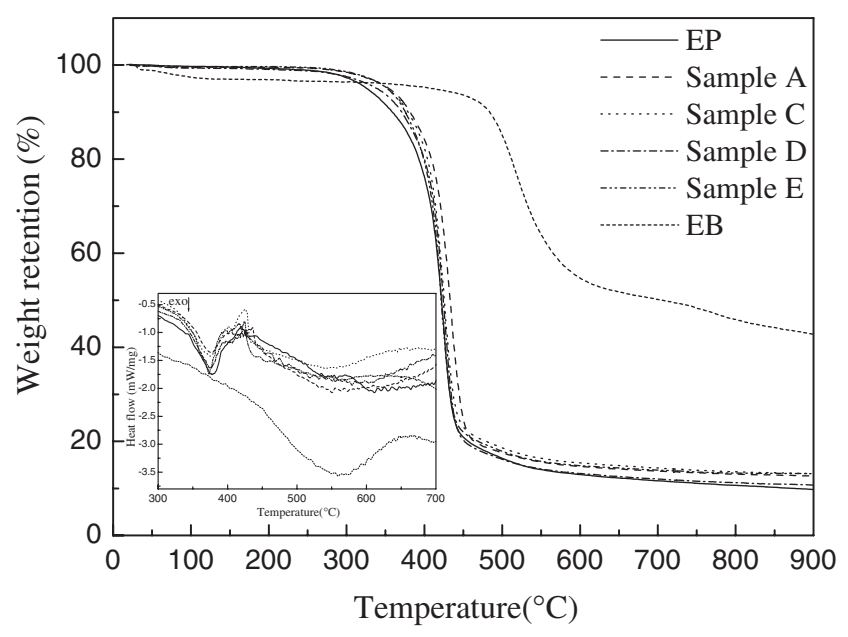

Figure 6. Thermograms of EB and EP/EB particles.

For the reason of no acidic dopant, EB keeps constant weight till ca. $400{ }^{\circ} \mathrm{C}$ and then comes the second weight loss from 400 to $600^{\circ} \mathrm{C}$ which is caused by the thermal decomposition of PANI backbone chains, ${ }^{22}$ and which can also be testified from the corresponding DSC curves (inset of Figure 6). EP maintains nearly the same weight below $300^{\circ} \mathrm{C}$ and this can be related to its rigid backbone chain. A remarkable weight loss between $300-500^{\circ} \mathrm{C}$ for EP comes from the decomposition of the backbone chain, which is in accordance with the relevant DSC curves (insets of Figure 6). All EP/EB particles exhibits a similar thermal behavior with that of EP, that is a remarkable weight loss in TGA curves and a large heat flow in their corresponding DSC curves, which is primarily caused by the extremely low EB loading in EP/EB particles.

\section{Passivation Effect of the EB Coatings}

By now, it was universally agreed that PANI was a good corrosion inhibitor. However, the solution based ways made the PANI containing coating costly. Dispersion way was then deemed an effective for PANI coating preparation. Differing from Wessling's "dispersion", 23,24 we prepared dispersion based PANI anti-corrosion coating by a direct ball milling of EB particles and EP with the help of MIBK in a earlier paper $^{25}$ and found that (1) EB's oxidation-reduction property still performs in cured epoxy coating, and (2) a minimum loading of $\mathrm{EB}$, which is $10 \mathrm{wt} \%$, was needed to achieve good anti-corrosive effect.

In $3.0 \mathrm{wt} \%$ aqueous $\mathrm{NaCl}$, corrosion potentials of epoxy coated coupons decreased to a constant value slightly higher than that of bare CRS (which is $-649 \mathrm{mV}$ vs. $\mathrm{Ag} / \mathrm{AgCl}$ ) in $48 \mathrm{~h}$, which was resulted from the physical barrier property of the coating. The behavior of corrosion potential to immersing time of EB coating was similar to that of pure epoxy coating in case of EB loading less than $1.27 \mathrm{wt} \%$ in EB coating, indicating of ineffective anti-corrosive property. While in case of EB coatings with EB loading larger than $1.27 \mathrm{wt} \%$, corrosion potentials decreased slowly with immersion time and stabilized at a higher value of $-433 \mathrm{mV}$ ( $v s . \mathrm{Ag} / \mathrm{AgCl}$ ), which was equivalent to that of the EB passivated CRS. Also the corrosion potentials decrease slower than that of directly ball milled EB containing coating (Figure 7).

The diameters of EB particles range from $30 \mathrm{~nm}$ to $200 \mathrm{~nm}$ and with the most of $100 \mathrm{~nm}$ in the directly ball milled EB containing coatings, while in EB coatings, the diameters of EB particles were in the range of 20-50 nm (Figure 8), which were smaller and more uniform than that of the directly ball milled ones. Accordingly, EB coating exhibit better corrosion property than the directly ball milled one with lower EB loading. The crystallograms of the EB coating shown

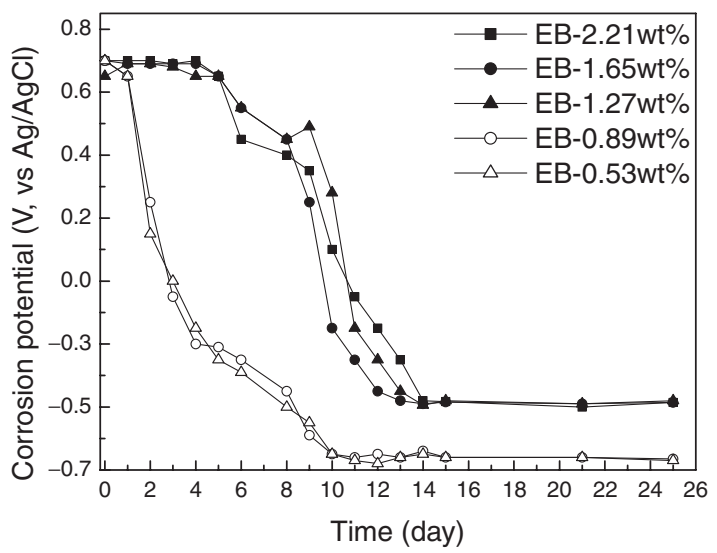

Figure 7. Plots of corrosion potential against Immersion time in $3.0 \mathrm{wt} \%$ aqueous $\mathrm{NaCl}$. 


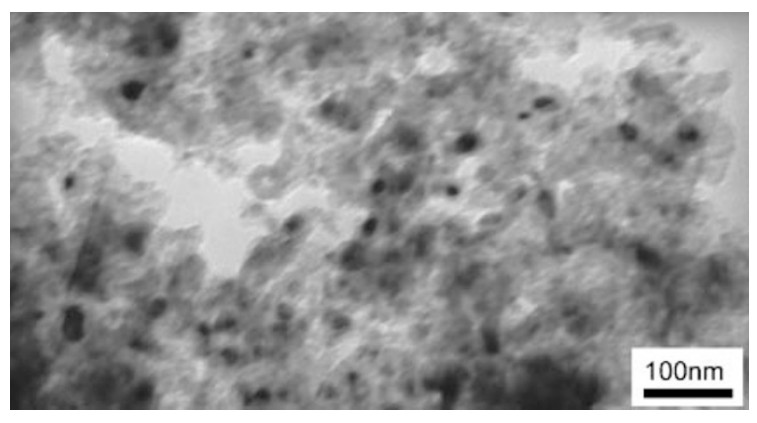

Figure 8. Size of EB particles in EB coatings (TEM).

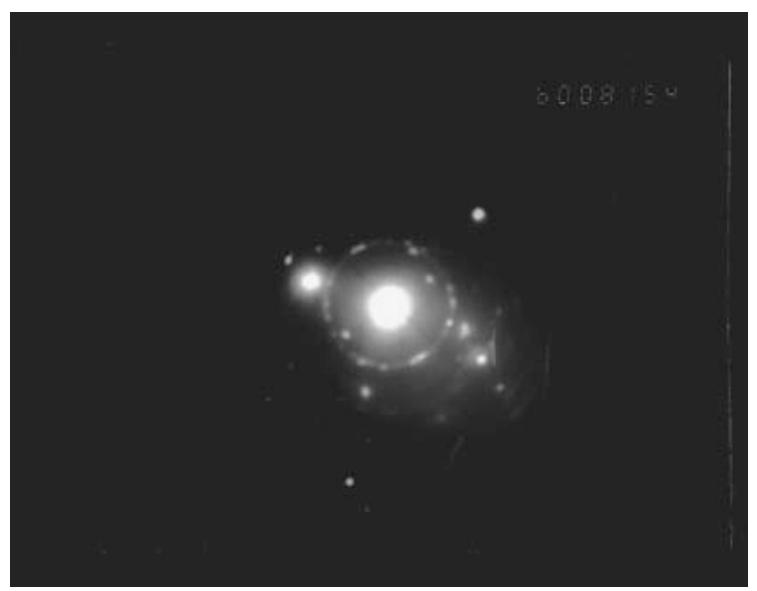

(A)

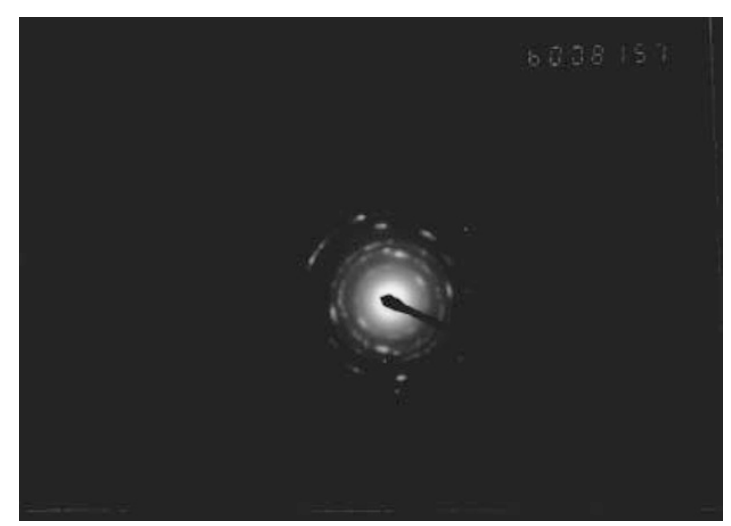

(B)

Figure 9. Crystallograms of the EB coating (TEM).

in Figure 9A and B indicate that the coating exhibits, to some extent, metallic properties, which made it easy to ennoble the metal surface according to Wessling. ${ }^{23}$

\section{CONCLUSIONS}

In conclusion, EB coating made from EP/EB particles, which were prepared by chemical oxidation of ANI with the presence of EP particles, provides excellent anti-corrosive property on CRS in extremely low EB loading compared with that of directly ball milled EB containing coating. The oxidation state of EB molecules in EP/EB particles decreased with the increase of ANI/EP in the reactants. Hydrogen bonding was found between EB and EP molecules. Thermal behavior of $\mathrm{EP} / \mathrm{EB}$ particles was similar to that of EP due to the extremely low EB contents of the particles.

Acknowledgment. The research is supported by the National Natural Science Foundation of China (No. 59903005).

\section{REFERENCES}

1. V. G. Kulkarni, Synth. Met., 71, 2129 (1995).

2. D. C. Trivedi and S. K. Dhawan, Polym. Adv. Technol., 4, 335 (1993).

3. O. T. Ikkala, L. O. Pietilä, P. Passiniemi, T. Vikki, H. Österholm, L. Ahjopalo, and J. E. Österholm, Synth. Met., 84, 55 (1997).

4. F. Hide, L. Diaz-Garcia, B. Schwartz, M. Andersson, Q. Pei, and A. Heeger, Science, 273, 1833 (1996).

5. R. B. Kaner, Synth. Met., 125, 65 (2002).

6. S. K. Dhawan and D. C. Trivedi, Polym. Int., 25, 55 (1991).

7. D. C. Trivedi and S. K. Dhawan, Synth. Met., 58, 309 (1993).

8. S. K. Dhawan and D. C. Trivedi, J. Polym. Sci., 58, 815 (1995).

9. X. Jing, W. Zhao, and L. Lan, J. Mater. Sci. Lett., 19, 377 (2000).

10. S. Ross and I. Morrison, in "Colloidal Systems and Interfaces,” John Wiley \& Sons, Inc., New York, N.Y., 1988, p. 59.

11. B. Wessling, Z. Phys. Chem., 191, 119 (1995).

12. J. L. Forveille and L. Olmedo, Synth. Met., 65, 5 (1994).

13. E. M. Genies, C. Petrescu, and L. Olmedo, Synth. Met., 41, 665 (1991).

14. J. B. Jun, J. W. Kim, and K. D. Suh, Macromol. Chem. Phys., 203, 1011 (2002).

15. I. Sapurina, A. Riede, and J. Stejskal, Synth. Met., 123, 503 (2001).

16. R. Mazeikiene and A. Malinauskas, Synth. Met., 108, 9 (2000).

17. H. L. Frisch, B. W. Xi, Y. C. Qin, M. Rafailovich, N. L. Yang, and X. Z. Yan, High Performance Polym., 12, 543 (2000).

18. X. Jing, Q. Yang, and M. Zheng, Chin. Synth. Resin Plast., 18, 10 (2001).

19. Y. Cao, Synth. Met., 35, 319 (1990).

20. Z. F. Li, E. T. Kang, K. G. Neoh, and K. L. Tan, Synth. Met., 87, 45 (1997).

21. J. Yue, A. J. Epstein, Z. Zhong, and P. K. Gallagher, Synth. Met., 41, 765 (1991).

22. Y. Wei and K. F. Hsueh, J. Polym. Sci., Part A: Polym. Chem., 27, 4351 (1989).

23. B. Wessling, Synth. Met., 93, 143 (1998).

24. B. Wessling, Chem. Innovat., 31, 34 (2001).

25. X. Jing, Y. Wang, and J. Qiang, J. Chin. Soc. Corro. Protec., in press. 